\title{
Distributed Fuzzy Adaptive Control for Heterogeneous Nonlinear Multiagent Systems with Similar Composite Structure
}

\author{
Yongqing Fan (D), Tiantian Xiao, and Zhen Li \\ School of Automation, Xi'an Key Laboratory of Advanced Control and Intelligent Process, \\ Xi'an University of Posts and Telecommunications, Xi'an 710121, China
}

Correspondence should be addressed to Yongqing Fan; fanyongqing@xupt.edu.cn

Received 3 April 2020; Accepted 7 May 2020; Published 1 June 2020

Guest Editor: Yan Wu

Copyright ( 92020 Yongqing Fan et al. This is an open access article distributed under the Creative Commons Attribution License, which permits unrestricted use, distribution, and reproduction in any medium, provided the original work is properly cited.

A distributed fuzzy adaptive control with similar parameters is constructed for a class of heterogeneous multiagent systems. Unlike many existing works, the dimensions of each multiagent dynamic system are considered to be nonidentical in this paper. Firstly, similar properties for different dimensions of multiagent systems are introduced, and some similar parameters among multiagent systems are also proposed. Secondly, a distributed fuzzy adaptive control on the basis of similar parameters is designed for the consensus of leader-follower multiagent systems. Following the graph theory and Lyapunov stability approach, it is concluded that UUB (uniformly ultimately bounded) of all signals in the closed-loop system can be guaranteed, and the consensus tacking error converges to a small compact zero set. Finally, a simulation example with different dimensions is provided to illustrate the effectiveness of the proposed method.

\section{Introduction}

Multiagent systems have been widely utilized in various fields such as remedial actions [1, 2], social engineering systems [3], satellites engineering [4], and robots cooperative $[5,6]$. More and more researchers inclined to design the fundamental collective controls for multiagent systems to make sure that the consensus or synchronization of leader-follower can be guaranteed, and many excellent controls for linear and nonlinear multiagent systems were proposed in recent years [7-10]. Generally speaking, the main work in designing controls is that all agents in the entire dynamic network must reach an agreement, and the information of each agent only can be shared locally. Unfortunately, in lots of actual engineering systems, uncertain nonlinear components existed such as electrical control systems and mechanical control systems; hence, it is a challenge to project appropriate control with limited information.

Fuzzy logic system (FLS) and neural network (NN) are two universal approximations to compensate uncertain terms in all sorts of complexity fields [11-15], and a great quantity of corresponding research works was derived by scholars [16-21]. For example, aiming at the high-order multiagent systems with unknown nonlinearities in [22], an observer-based distributed fuzzy adaptive control was designed to deal with the unknown nonlinear functions. For a class of strict feedback form of multiagent systems, a novel event-triggered control was presented for the consensus tracking in [23]. Adaptive NN event-triggered control plan was investigated for the nonstrict feedback multiagent systems with sensor faults and input saturation in [24]. In [25], a fuzzy observer was designed to evaluate the unmeasurable states of nonlinear multiagent systems, and an event-triggered control approach was studied to make the followers synchronize with leader's trajectory. However, these existing results only researched on the identity of each agent, which means that the states of every agent have same dynamical behaviors [22-25]. In order to break this limitation, different dynamic behaviors of each agent that can be called as heterogeneous multiagent systems have been studied [26-28]. For instance, a distributed adaptive fuzzy control combining with the backstepping technique was addressed for a class of second-order heterogeneous multiagent systems in [29]. In [30], the output consensus of multiagent systems was guaranteed by using the devised 
fuzzy adaptive control. A robust consensus protocol was designed for essential heterogeneous multiagent systems in [31]. In order to ensure the consensus of heterogeneous multiagent systems, a distributed proportional integral control based on sufficient conditions was derived in [32]. It should be noted that the proposed control schemes in $[8,10,26-28]$ were only valid for linear multiagent systems. These abundant research achievements provided well guidance for some new design algorithm controls of heterogeneous multiagent systems. Nevertheless, the dimensions of every agent are completely congruent in these literatures [8, 26-32], and the raised control schemes will be invalided to settle the consensus or synchronization of multiagent systems with different dimensions. Consequently, it is necessary to exploit other original control approaches to tackle the consensus of multiagent systems with distinct dimensions.

Motivated by the similar properties of large-scale systems in [33-38], the definition of similar nodes was introduced for large-scale composite systems with different dimensions, and some effective controls with similar parameters were addressed. From the viewpoint of mathematics, every agent can be defined as a series of nodes in a network; hence, the character of similar nodes in these excellent research works can be drawn to develop consensus control with similar parameters.

This paper attempts to investigate a novel consensus fuzzy adaptive control for a class of multiagent systems with different dimensions, in which the dimensions of each agent are unequal, and the similar parameters of agents are used for devising consistency control. Compared to recent existing works on the consensus of heterogeneous multiagent systems, the principal contributions are three aspects: first, the dimensions of follower systems are different with the dimensions of leader, and the similar definition among multiagent systems is explored. Second, a distributed fuzzy adaptive control methodology with similar parameters is provided. Last, the control matrix gain can be solved by the condition of proposed linear matrix inequality (LMI).

The remaining parts of this paper are organized as follows. Interaction topology, the property of similar composite structure, and FLS are displayed in Section 2. Section 3 presents the fuzzy adaptive control and stability analysis. A simulation example is given for the consensus of multiagent system with nonidentical dimension in Section 4. Finally, Section 5summarizes conclusions.

Throughout this paper, the following notations are hired. $R^{n \times n}$ denotes the $n \times n$ dimensional Euclidean space; $\operatorname{diag}\left\{A_{1} \quad A_{2} \cdots A_{N}\right\}$ expresses the block-diagonal matrix with matrices $A_{1} \quad A_{2} \quad \cdots \quad A_{N}$ on its principal diagonal; the notation $\|\cdot\|$ refers to the vector-2-norm. $A^{\mathrm{T}}$ and $A^{-1}$ denote the transpose matrix and inverse matrix of $A$, respectively; $I_{n}$ represents the identity matrix with $n$ appropriate dimensions; and $P>0(<0)$ means that $P$ is a positive (negative) definite matrix. The Kronecker product of matrices $A$ and $P$ is symbolized by $A \otimes P$; the maximum and minimum eigenvalues matrix $A$ are denoted corresponding to $\lambda_{\max }(A)$ and $\lambda_{\min }(A)$.

\section{Preliminaries and Problem Formulation}

2.1. Graph Theory. A directed digraph $G=\{V, E\}$ is utilized to describe the information exchange among each agent, where $V=\left[\begin{array}{llll}v_{1} & v_{2} & \cdots & v_{N}\end{array}\right]$ stands for the nonempty set of nodes for each agent. The edge set $E$ contains an edge $\left(v_{j}, v_{i}\right)$ which means node $v_{j}$ is able to transfer the relative state information to node $v_{i}$; then, nodes $i$ and $j$ are called as the neighbors when the edge $\left(v_{j}, v_{i}\right)$ exists. Let $N_{i}$ denote the set of neighbors of node $i(i=1,2, \ldots, N)$. The directed digraph $G$ can also be described by an adjacency matrix $\left[\alpha_{i j}\right]$, where $\alpha_{i j}=\left\{\begin{array}{ll}1, & \left(v_{j}, v_{i}\right) \in E \\ 0, & \text { otherwise }\end{array}\right.$. In addition, it is assumed that there are no repeated edges and no self-loops, i.e., $\alpha_{i i}=0$. The Laplacian matrix $L=\left[l_{i j}\right] \in R^{N \times N}$ is defined as $l_{i j}=\left\{\begin{array}{ll}-\alpha_{i j}, & i \neq j \\ \sum_{k \neq i, k=1}^{N} \alpha_{i k}, & i=j\end{array}\right.$.

A digraph is said to have a spanning tree, if there exists a node that is called as the root such that the node has directed paths to all other nodes in the graph. The graph $\bar{G}$ consists of $G$, node 0 (the leader), and the directed edges from the node 0 to the followers in $G$, and only a small percentage of the followers can receive the information from the leader. Then, we get the following lemma.

Lemma 1 (see [8]). Let the matrix $\bar{L}=L+\operatorname{diag}\left(\left[\begin{array}{llll}\alpha_{10} & \alpha_{20} & \cdots & \alpha_{N 0}\end{array}\right]\right)$ is positive definite with $\alpha_{i 0}>0$, the ith agent has access to the leader's state information, whereas $\alpha_{i 0}=0$ if otherwise.

2.2. Preliminaries and Multiagent System. Consider a group of agent system with a leader and $N$ followers labeled as 0 and $1,2, \ldots, N$, respectively. The dynamics of the leader is described as

$$
\dot{x}_{0}(t)=A_{0} x_{0}(t)+B_{0}\left[u_{0}(t)+s\left(x_{0}, t\right)\right],
$$

where $A_{0}(t) \in R^{n_{0} \times n_{0}}$ and $B_{0} \in R^{n_{0} \times m_{0}}$ are the system matrix and input matrix of leader, respectively. $x_{0}(t) \in R^{n_{0} \times 1}$ is the state vector of leader. $u_{0}(t)$ denotes the input vector of leader, and matrix $K_{0} \in R^{m_{0} \times n_{0}}$ will be given in the process of control design, which will make $A_{0}+B_{0} K_{0}$ be Hurwitz stabilized. $s\left(x_{0}, t\right)$ is defined as an input bounded signal and satisfies $\left|s\left(x_{0}, t\right)\right| \leq \bar{s}$ for all $t \geq t_{0}$, and $\bar{s}$ is a known constant.

The dynamics of the followers are defined as follows:

$$
\dot{x}_{i}(t)=A_{i} x_{i}(t)+B_{i}\left[u_{i}(t)+g_{i}\left(x_{i}\right)\right], \quad i=1,2, \ldots, N,
$$

where $A_{i} \in R^{n_{i} \times n_{i}}$ and $B_{i} \in R^{n_{i} \times m_{i}}$ denote the system matrix and input matrix in the $i$ th followers system, respectively. $x_{i}(t) \in R^{n_{i} \times 1}$ and $u_{i}(t) \in R^{m_{i} \times n_{i}}$ are corresponding to the state vector and input vector of the $i$ th follower, respectively. $g_{i}\left(x_{i}\right)$ represents the unknown nonlinear function.

Assumption 1 (see [38]). Consider $N$ agent systems as given in (2), and the follower system (2) is called similar to the leader system (1), if there exists $N$ matrices $K_{i} \in R^{m_{i} \times n_{i}}$, 
matrix $K_{0} \in R^{m_{0} \times n_{0}}$, and $N$ matrices $T_{i} \in R^{n_{0} \times n_{i}}$ satisfying the following condition:

$$
\left\{\begin{array}{l}
T_{i}\left(A_{i}+B_{i} K_{i}\right)=\left(A_{0}+B_{0} K_{0}\right) T_{i}, \\
T_{i} B_{i}=B_{0} .
\end{array}\right.
$$

Definition 1. In Assumption 1, $T_{i}$ and $K_{i}$ and $K_{0}$ are called as similar parameters with different dimensions.

Remark 1. Assumption 1 ensures that the matrices $A_{i}+$ $B_{i} K_{i}$ and $A_{0}+B_{0} K_{0}$ possess some common eigenvalues. Thus, Assumption 1 implies that the agent systems as given in (1) and (2) contain certain similar inner dynamical behavior, and these agent systems are named as similar structure agents with similar parameters.

Remark 2. From a mathematical point of view, Assumption 1 admits that the state dimensions can be different or identical in multiagent systems. Especially, if $A_{i}=A_{0}$ and $n_{i}=n_{0}$ in (1) and (2), then the agent system (1) and (2) coincides with the system in [26-32].

In order to address the unknown nonlinear function, the following fuzzy logic system (FLS) is utilized in this paper. It mainly includes four parts: fuzzifier, fuzzy rule base, fuzzy inference engine, and defuzzifier. The fuzzifier is a mapping from the input space and state space to the fuzzy sets. The fuzzy rule base contains several linguistics rules. The fuzzy rules are represented as follows:

$$
p \text { th: If } x_{1} \text { is } F_{1}^{p}, x_{2} \text { is } F_{2}^{p}, \ldots, x_{n} \text { is } F_{n}^{p} \text {, then } y \text { is } G_{p}, \quad p=1,2, \ldots, h \text {, }
$$

where $x=\left(x_{1}, x_{2}, \ldots, x_{n}\right)^{\mathrm{T}}$ and $y$ are the input and output of the FLS, respectively. $\mu_{F_{i}^{p}}\left(x_{i}\right)$ and $\mu_{G_{p}}(y)$ are the membership functions of fuzzy sets $F_{i}^{p}$ and $G_{p}$, respectively. By employing singleton fuzzifier, center average defuzzifier, and product inference, the output of FLS can be expressed as

$$
y(x)=\frac{\sum_{p=1}^{h} \theta_{p} \prod_{i=1}^{n} \mu_{F_{i}^{p}}\left(x_{i}\right)}{\sum_{p=1}^{h}\left[\prod_{i=1}^{n} \mu_{F_{i}^{p}}\left(x_{i}\right)\right]}
$$

where $\theta_{p}=\arg \max _{y \in R} \mu_{G_{p}}(y)$. If we denote $\theta^{\mathrm{T}}=$ $\left[\theta_{1}, \theta_{2}, \ldots, \theta_{h}\right]$ and $\psi(x)=\left[\psi_{1}(x), \psi_{2}(x), \ldots, \psi_{n}(x)\right]^{\mathrm{T}}$, then the fuzzy logical systems can be rewritten as

$$
y(x)=\theta^{\mathrm{T}} \psi(x) .
$$

Lemma 2 (see [39]). For any given two vectors $x, y \in R^{n}$ and a scalar $a>0$, the following inequality holds:

$$
2 x^{\mathrm{T}} y \leq a x^{\mathrm{T}} x+a^{-1} y^{\mathrm{T}} y .
$$

Lemma 3 (see [11]). For any given uncertain continuous function $g(x)$ on a compact set $\Omega$ and an arbitrary approximation accuracy $\varepsilon>0$, there exists a FLS such as (4) such that the following universal approximation holds:

$$
\sup _{x \in \Omega}\left|\theta^{\mathrm{T}} \psi(x)-g(x)\right| \leq \varepsilon .
$$

According to Lemma 3, we know that the unknown nonlinear function $g_{i}\left(x_{i}\right)$ in the $i$ th follower system can be approximated by

$$
g_{i}\left(x_{i}\right)=\theta_{i}^{\mathrm{T}} \psi_{i}\left(x_{i}\right)+\varepsilon_{i}(t),
$$

where $\theta_{i}(t)=\left[\theta_{i 1}(t), \theta_{i 2}(t), \ldots, \theta_{i m_{i}}(t)\right]^{\mathrm{T}}$ is unknown parameter vector that will be designed by adaptive laws and $\psi_{i}\left(x_{i}\right)=\left[\psi_{i 1}, \psi_{i 2}, \ldots, \psi_{i m_{i}}\right]^{\mathrm{T}}$ is the fuzzy basis function as shown in (6). In this paper, approximation accuracy $\varepsilon_{i}(t)$ is a time-varying function and satisfies $\left|\varepsilon_{i}(t)\right| \leq \bar{\varepsilon}_{i}$ for all $t \geq t_{0}$, where $\bar{\varepsilon}_{i}$ is a known constant.

Control Purpose. The aim of this paper is to design a distributed fuzzy adaptive control by using similar parameter such that the consensus errors are UUB.

\section{Main Results}

To solve the consensus problem of the leader-follower system (1) and (2), the following control strategy is proposed:

$$
\begin{aligned}
u_{i}(t)= & c F \sum_{j=1}^{n} \alpha_{i j}\left(T_{i} x_{i}(t)-T_{j} x_{j}(t)\right)+K_{i} x_{i}(t) \\
& +\bar{K}\left(T_{i} x_{i}(t)-T_{0} x_{0}(t)\right)-\bar{\theta}_{i}^{\mathrm{T}}(t) \psi_{i}\left(x_{i}\right),
\end{aligned}
$$

where $F=-0.5 B_{0}^{\mathrm{T}} P$ and the control gain matrix $\bar{K}=Y X^{-1}$ can be proposed by solving the following LMI:

$$
\left[\begin{array}{cc}
\Delta & X^{T} \\
* & -Q
\end{array}\right] \leq 0
$$

where $\Delta=A_{0} X+X A_{0}^{T}+\bar{B}_{0} X+X \bar{B}_{0}^{T}+B_{0} Y+Y^{T} B_{0}^{T}-c \lambda B_{0}$ $B_{0}^{T}, X>0, Q>0$, and parameter $0<\lambda<\lambda_{\text {min }}(\bar{L})$.

In control (10), parameters $\bar{\theta}_{i}(t)$ denote the estimation values of $\theta_{i}(t)$ and $\theta_{i}(t)$ are their errors, and the relation between them is defined as $\widetilde{\theta}_{i}(t)=\bar{\theta}_{i}(t)-\theta_{i}(t)$. The estimation $\bar{\theta}_{i}(t)$ can be designed as

$$
\dot{\bar{\theta}}_{i}(t)=-\kappa_{\theta_{i}} \bar{\theta}_{i}(t)+\rho_{\theta_{i}} \psi_{i}\left(x_{i}\right)\left(P B_{0}\right)^{\mathrm{T}} e_{i}(t),
$$

where $\kappa_{\theta_{i}}=\left[\begin{array}{llll}\kappa_{\theta_{i 1}} & \kappa_{\theta_{i 2}} & \cdots & \kappa_{\theta_{i m_{i}}}\end{array}\right]$ is a vector consisting of some known positive constants given by designer, $\theta_{i}(t)=\left[\theta_{i 1}^{T}(t), \theta_{i 2}^{T}(t), \ldots, \theta_{i m_{i}}^{T}(t)\right]^{T}, \rho_{\theta_{i}}>0$.

Theorem 1. Suppose that Assumption 1 is satisfied, and at least one agent system in connected graph $G$ has access to the state information of the leader system (1). With the action of control (10), the consensus error between leader system (1) and follower system (2) is UUB and belongs to the following set: 


$$
\begin{aligned}
D & :=\left\{e(t)=\left[e_{1}^{\mathrm{T}}(t) \quad e_{2}^{\mathrm{T}}(t) \cdots e_{N}^{\mathrm{T}}(t)\right]^{\mathrm{T}}:\|e(t)\|\right. \\
& \left.\leq \sqrt{\frac{\sigma}{\lambda_{\min }\left(I_{N} \otimes P\right) \gamma}}\right\},
\end{aligned}
$$

where $\sigma$ and $\gamma$ will be given later.

Proof. Let consensus error as $e_{i}(t)=T_{i} x_{i}(t)-T_{0} x_{0}(t)$ and $T_{0}=I^{n_{0} \times n_{0}}$ is an identity matrix. By applying Assumption 1, it becomes

$$
\begin{aligned}
T_{i} \dot{x}_{i}(t)= & \left(A_{0}+B_{0} K_{0}\right) T_{i} x_{i}(t)-B_{0} \bar{\theta}_{i}^{\mathrm{T}}(t) \psi_{i}\left(x_{i}\right) \\
& +B_{0} g_{i}(x)+c \sum_{j=1}^{n} \alpha_{i j} B_{0} F\left(T_{i} x_{i}(t)-T_{j} x_{j}(t)\right) \\
& +B_{0} \bar{K} e_{i}(t) .
\end{aligned}
$$

The leader system is transformed as

$$
\begin{aligned}
T_{0} \dot{x}_{0}(t)= & \left(A_{0}+B_{0} K_{0}\right) T_{0} x_{0}(t) \\
& +c \alpha_{i 0} B_{0} F\left(T_{i} x_{i}(t)-T_{0} x_{0}(t)\right)+B_{0} s\left(x_{0}, t\right),
\end{aligned}
$$

and then, the error system can be transformed as

$$
\begin{aligned}
\dot{e}_{i}(t)= & \left(A_{0}+B_{0} K_{0}+B_{0} \bar{K}\right) e_{i}(t) \\
& +B_{0}\left[-\widetilde{\theta}_{i}^{\mathrm{T}}(t) \psi_{i}\left(x_{i}\right)+\varepsilon_{i}(t)+s\left(x_{0}, t\right)\right] \\
& +c B_{0} F\left\{\sum_{j=1}^{n} \alpha_{i j}\left[e_{i}(t)-e_{j}(t)\right]\right. \\
& \left.-\alpha_{i 0}\left[T_{i} x_{i}(t)-T_{0} x_{0}(t)\right]\right\} .
\end{aligned}
$$

For brevity, (16) is equal to

$$
\begin{aligned}
\dot{e}(t) & =\left[I_{N} \otimes\left(A_{0}+B_{0} K_{0}+B_{0} \bar{K}\right)-(c \bar{L}) \otimes\left(B_{0} F\right)\right] e(t) \\
& +\left(I_{N} \otimes B_{0}\right)\left[-\tilde{\theta}^{\mathrm{T}}(t) \psi(x)+\varepsilon(t)+s\left(x_{0}, t\right)\right],
\end{aligned}
$$

where $e(t)=\left[e_{1}^{T}(t), e_{2}^{T}(t), \ldots, e_{N}^{T}(t)\right]^{T}, \quad \tilde{\theta}(t)=\left[\tilde{\theta}_{1}^{T}(t)\right.$, $\left.\tilde{\theta}_{2}^{T}(t), \ldots, \widetilde{\theta}_{N}^{T}(t)\right]^{T}, \quad \psi(x)=\left[\psi_{1}^{T}\left(x_{1}\right), \psi_{2}^{T}\left(x_{2}\right), \ldots, \psi_{N}^{T}\right.$ $\left.\left(x_{N}\right)\right]^{T}, \quad \varepsilon(t)=\left[\varepsilon_{1}^{T}(t), \varepsilon_{2}^{T}(t), \ldots, \varepsilon_{N}^{T}(t)\right]^{T}, \quad$ and $-\bar{L} e(t)=$ $\sum_{j=1}^{n} \alpha_{i j}\left[e_{i}(t)-e_{j}(t)\right]-\alpha_{i 0}\left[T_{i} x_{i}(t)-T_{0} x_{0}(t)\right]$

The following candidate Lyapunov function is considered:

$$
V(t)=\frac{1}{2} e^{\mathrm{T}}(t)\left(I_{N} \otimes P\right) e(t)+\frac{1}{2} \widetilde{\theta}^{\mathrm{T}}(t) \rho_{\theta}^{-1} \widetilde{\theta}(t) .
$$

The derivative of $V(t)$ along system (17) is

$$
\begin{aligned}
\dot{V}(t)= & \frac{1}{2} e^{\mathrm{T}}(t)\left\{I _ { N } \otimes \left[P\left(A_{0}+B_{0} K_{0}+B_{0} \bar{K}\right)\right.\right. \\
& \left.\left.+\left(A_{0}+B_{0} K_{0}+B_{0} \bar{K}\right)^{\mathrm{T}} P\right]-(c \bar{L}) \otimes\left(P B_{0} B_{0}^{\mathrm{T}} P\right)\right\} e(t) \\
& +\widetilde{\theta}^{\mathrm{T}}(t) \rho_{\theta}^{-1} \dot{\bar{\theta}}(t)+e^{\mathrm{T}}(t)\left[I_{N} \otimes\left(P B_{0}\right)\right] \\
& +\left[-\widetilde{\theta}^{\mathrm{T}}(t) \psi(x)+\varepsilon(t)+s\left(x_{0}, t\right)\right] \\
\leq & \frac{1}{2} e^{\mathrm{T}}(t)\left\{I _ { N } \otimes \left[P\left(A_{0}+B_{0} K_{0}+B_{0} \bar{K}\right)\right.\right. \\
& \left.\left.+\left(A_{0}+B_{0} K_{0}+B_{0} \bar{K}\right)^{\mathrm{T}} P\right]-c \lambda\left(P B_{0} B_{0}^{\mathrm{T}} P\right)\right\} e(t) \\
& +e^{\mathrm{T}}(t)\left[I_{N} \otimes\left(P B_{0}\right)\right][\varepsilon(t)+\bar{s}]-\frac{\kappa_{\theta} \widetilde{\theta}^{\mathrm{T}}(t) \bar{\theta}(t)}{\rho_{\theta}} \\
& +e^{\mathrm{T}}(t)\left[I_{N} \otimes\left(P B_{0}\right)\right][\varepsilon(t)+\bar{s}] . \\
= & -\frac{1}{2} e^{\mathrm{T}}(t)\left(I_{N} \otimes Q\right) e(t)-\frac{\kappa_{\theta}}{\rho_{\theta}} \widetilde{\theta}^{\mathrm{T}}(t) \bar{\theta}(t) \\
&
\end{aligned}
$$

Based on Lemma 3, one obtains that

$$
\begin{aligned}
-\tilde{\theta}^{\mathrm{T}}(t) \bar{\theta}(t) & =-\tilde{\theta}^{\mathrm{T}}(t)(\tilde{\theta}(t)+\theta(t)) \\
& \leq-\frac{1}{2} \tilde{\theta}^{\mathrm{T}}(t) \tilde{\theta}(t)+\frac{1}{2} \theta^{\mathrm{T}}(t) \theta(t) .
\end{aligned}
$$

Combining with (19) and (20), it becomes

$$
\begin{aligned}
\dot{V}(t) \leq & -\frac{1}{2} e^{\mathrm{T}}(t)\left(I_{N} \otimes Q\right) e(t)-\frac{1}{2} \frac{\kappa_{\theta}}{\rho_{\theta}} \widetilde{\theta}^{\mathrm{T}}(t) \tilde{\theta}(t) \\
& +\frac{1}{2} \frac{\kappa_{\theta}}{\rho_{\theta}} \theta^{\mathrm{T}}(t) \theta(t)+e^{\mathrm{T}}(t)\left[I_{N} \otimes\left(P B_{0}\right)\right][\varepsilon(t)+\bar{s}] \\
\leq & -\frac{1}{2} \lambda_{\min }\left(I_{N} \otimes Q\right) e^{\mathrm{T}}(t) e(t)-\frac{1}{2} \frac{\kappa_{\theta}}{\rho_{\theta}} \widetilde{\theta}^{\mathrm{T}}(t) \tilde{\theta}(t) \\
& +\|e(t)\| \cdot\left\|I_{N} \otimes\left(P B_{0}\right)\right\| \cdot(\bar{\varepsilon}+\bar{s})+\frac{1}{2} \frac{\kappa_{\theta}}{\rho_{\theta}} \theta^{\mathrm{T}}(t) \theta(t) \\
\leq & -\gamma V(t)+\sigma .
\end{aligned}
$$

If denoting $\gamma=\min \left\{\left(\lambda_{\min }\left(I_{N} \otimes Q\right) / \lambda_{\max }\left(I_{N} \otimes P\right)\right), \kappa_{\theta}\right\}$, $\sigma=\|e(t)\| \cdot\left\|I_{N} \otimes\left(P B_{0}\right)\right\| \cdot[\bar{\varepsilon}+\bar{s}]+(1 / 2)\left(\kappa_{\theta} / \rho_{\theta}\right) \theta^{\mathrm{T}}(t) \theta(t)$, then it follows 


$$
V(t) \leq\left[V(0)-\frac{\sigma}{\gamma}\right] e^{-\gamma t}+\frac{\sigma}{\gamma}
$$

Inequality (22) shows that the consensus error $e(t)$ can be guaranteed to be UUB with

$$
\|e(t)\| \leq \frac{1}{\sqrt{\lambda_{\min }\left(I_{N} \otimes P\right)}} \sqrt{\left[V(0)-\frac{\sigma}{\gamma}\right] e^{-\gamma t}+\frac{\sigma}{\gamma}} .
$$

Accordingly, the conclusion is

$$
\lim _{t \rightarrow \infty}\|e(t)\| \leq \sqrt{\frac{\sigma}{\lambda_{\min }\left(I_{N} \otimes P\right) \gamma}} .
$$

(24) means that the consensus error $e(t)$ converges to the set $D$, which is defined in (13). This completes the proof.

Remark 3. The inequality $P\left(A_{0}+B_{0} K_{0}+B_{0} \bar{K}\right)+\left(A_{0}+\right.$ $\left.B_{0} K_{0}+B_{0} \bar{K}\right)^{\mathrm{T}} P-c \lambda P B_{0} B_{0}^{T} P \leq-Q$ is a nonlinear matrix inequality; through multiplying by $P^{-1}$ on the left and right sides of this inequality and defining $B_{0} K_{0}=\bar{B}_{0}, P^{-1}=X$, and $Y=\bar{K} X$, the inequality (11) can be obtained.

The multiagent system with similar composite structure and proposed control scheme is explained as the block diagram in Figure 1

\section{Simulation Example}

In this section, a simulation example is given to prove the effectiveness of the proposed control. Six agent systems are considered including one leader labeled 0 and five followers labeled 1,2,3,4, and 5. Figure 2 shows the communication between the leader and each follower, it is easy to know that only the first agent can obtain the state information of the leader.

From Figure 2, the Laplacian matrix of the follower system and the degree matrix of the leader system can be calculated as follows:

$$
\begin{aligned}
L & =\left[\begin{array}{ccccc}
2 & -1 & -1 & 0 & 0 \\
-1 & 2 & 0 & -1 & 0 \\
-1 & 0 & 3 & -1 & -1 \\
0 & -1 & -1 & 2 & 0 \\
0 & 0 & -1 & 0 & 1
\end{array}\right], \\
\operatorname{diag}\left(\left[\begin{array}{lllll}
\alpha_{10} & \alpha_{20} & \cdots & \alpha_{50}
\end{array}\right]\right) & =\left[\begin{array}{ccccc}
1 & 0 & 0 & 0 & 0 \\
0 & 0 & 0 & 0 & 0 \\
0 & 0 & 0 & 0 & 0 \\
0 & 0 & 0 & 0 & 0 \\
0 & 0 & 0 & 0 & 0
\end{array}\right] .
\end{aligned}
$$
by

$$
A_{0}=\left[\begin{array}{cccccccc}
1 & -7 & -3 & -1 & -1 & 2 & -2 & -4 \\
5 & 2 & -4 & 2 & -7 & -8 & 9 & -1 \\
0 & 0 & -1 & 0 & 0 & 0 & 0 & 0 \\
0 & 0 & 0 & -2 & 0 & 0 & 0 & 0 \\
0 & 0 & 0 & 0 & -3 & 0 & 0 & 0 \\
0 & 0 & 0 & 0 & 0 & -6 & 0 & 0 \\
0 & 0 & 0 & 0 & 0 & 0 & -8 & 0 \\
0 & 0 & 0 & 0 & 0 & 0 & 0 & -9
\end{array}\right],
$$$$
A_{1}=\left[\begin{array}{ccccccc}
1 & -7 & -3 & -1 & -1 & 2 & -2 \\
2 & 7 & 3 & -2 & -8 & -10 & 2 \\
0 & 0 & -1 & 0 & 0 & 0 & 0 \\
0 & 0 & 0 & -2 & 0 & 0 & 0 \\
0 & 0 & 0 & 0 & -3 & 0 & 0 \\
0 & 0 & 0 & 0 & 0 & -6 & 0 \\
0 & 0 & 0 & 0 & 0 & 0 & -8
\end{array}\right] \text {, }
$$$$
B_{0}=\left[\begin{array}{c}
0 \\
1 \\
O_{1 \times 6}
\end{array}\right] \text {, }
$$$$
A_{2}=\left[\begin{array}{cccccc}
1 & -7 & -3 & -1 & -1 & 2 \\
3 & 7 & -2 & -1 & -6 & -9 \\
0 & 0 & -1 & 0 & 0 & 0 \\
0 & 0 & 0 & -2 & 0 & 0 \\
0 & 0 & 0 & 0 & -3 & 0 \\
0 & 0 & 0 & 0 & 0 & -6
\end{array}\right] \text {, }
$$

$$
B_{1}=\left[\begin{array}{c}
0 \\
1 \\
O_{1 \times 5}
\end{array}\right] \text {, }
$$$$
A_{3}=\left[\begin{array}{ccccc}
1 & -7 & -3 & -1 & -1 \\
5 & 3 & -5 & 2 & 3 \\
0 & 0 & -1 & 0 & 0 \\
0 & 0 & 0 & -2 & 0 \\
0 & 0 & 0 & 0 & -3
\end{array}\right],
$$$$
A_{4}=\left[\begin{array}{cccc}
1 & -7 & -3 & -1 \\
1 & 3 & 2 & 7 \\
0 & 0 & -1 & 0 \\
0 & 0 & 0 & -2
\end{array}\right] \text {, }
$$$$
A_{5}=\left[\begin{array}{ccc}
1 & -7 & -3 \\
5 & 2 & 1 \\
0 & 0 & -1
\end{array}\right] \text {, }
$$$$
B_{2}=\left[\begin{array}{lll}
0 & 1 & O_{1 \times 4}
\end{array}\right]^{\mathrm{T}} \text {, }
$$$$
B_{3}=\left[\begin{array}{lll}
0 & 1 & O_{1 \times 3}
\end{array}\right]^{\mathrm{T}} \text {, }
$$$$
B_{4}=\left[\begin{array}{lll}
0 & 1 & O_{1 \times 2}
\end{array}\right]^{\mathrm{T}} \text {, }
$$$$
B_{5}=\left[\begin{array}{lll}
0 & 1 & 0
\end{array}\right]^{\mathrm{T}} \text {. }
$$

By using the similar condition in Assumption 1, the similar parameters can be obtained as 


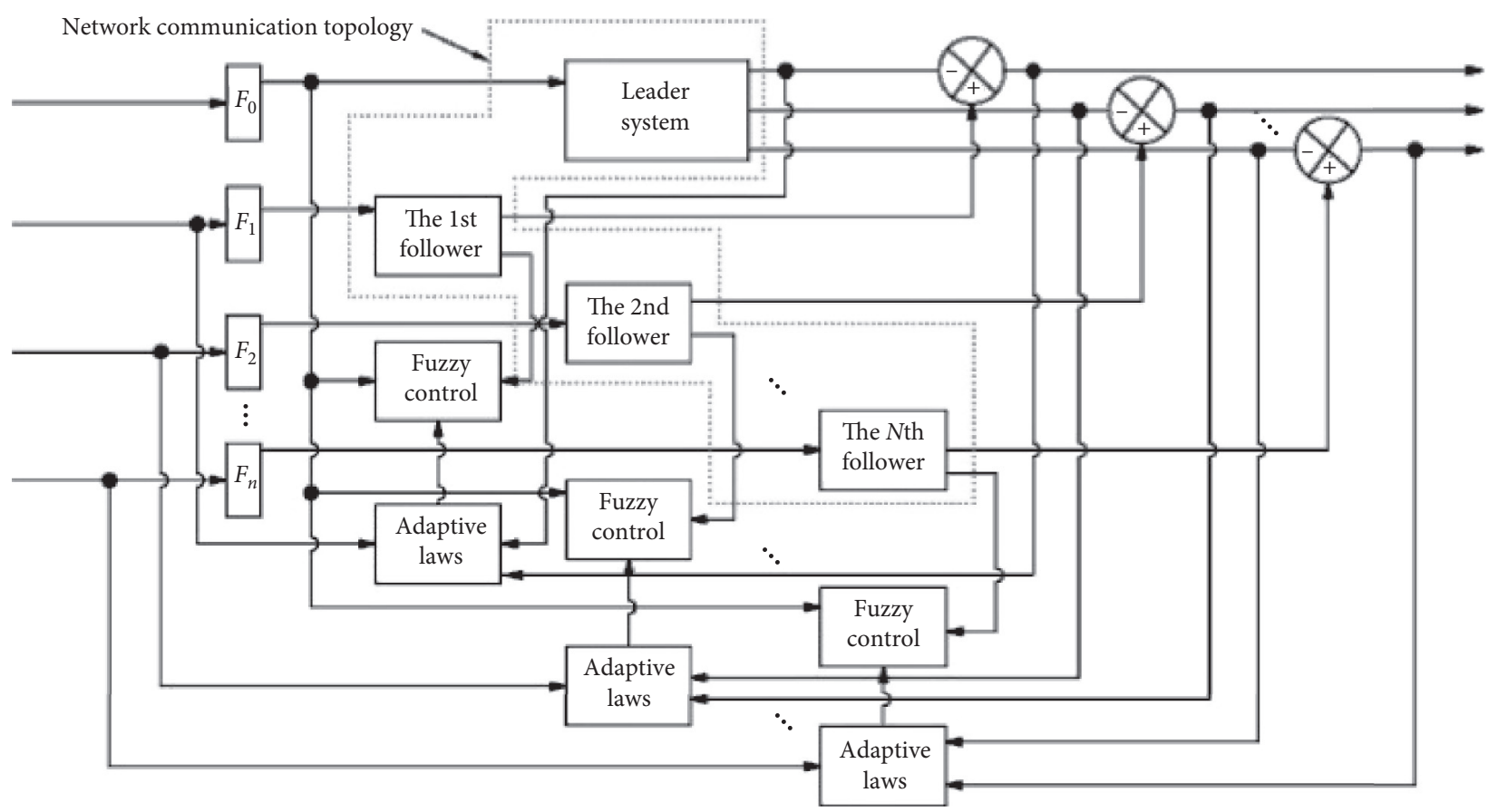

Figure 1: The block diagram of closed-loop multiagent systems.

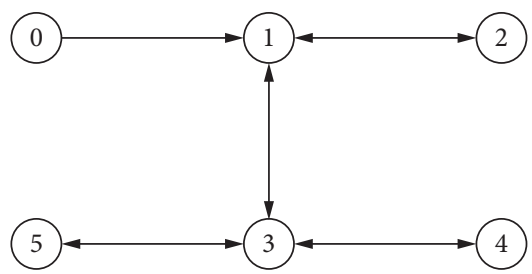

Figure 2: The communication topology.

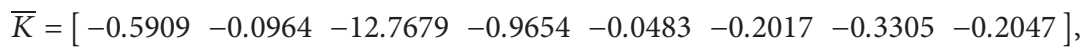

$$
\begin{aligned}
& P=\left[\begin{array}{cccccccc}
0.4057 & -0.1665 & -0.1051 & -0.0329 & -0.0324 & 0.0673 & -0.0684 & -0.1369 \\
-0.1665 & 0.4504 & 0.1632 & 0.0545 & 0.0553 & -0.1180 & 0.1225 & 0.2487 \\
-0.1051 & 0.1632 & 0.3894 & 0.0388 & 0.0379 & -0.0726 & 0.0708 & 0.1398 \\
-0.0329 & 0.0545 & 0.0388 & 0.3294 & 0.0120 & -0.0233 & 0.0229 & 0.0453 \\
-0.0324 & 0.0553 & 0.0379 & 0.0120 & 0.4195 & -0.0229 & 0.0226 & 0.0449 \\
0.0673 & -0.1180 & -0.0726 & -0.0233 & -0.0229 & 0.8276 & -0.0457 & -0.0914 \\
-0.0684 & 0.1225 & 0.0708 & 0.0229 & 0.0226 & -0.0457 & 1.1140 & 0.0923 \\
-0.1369 & 0.2487 & 0.1398 & 0.0453 & 0.0449 & -0.0914 & 0.0923 & 1.4012
\end{array}\right], \\
& T_{0}=I_{8} \text {, } \\
& T_{1}=\left[\begin{array}{ll}
I_{7} & O_{7 \times 1}
\end{array}\right]^{\mathrm{T}} \text {, } \\
& T_{2}=\left[\begin{array}{ll}
I_{6} & O_{6 \times 2}
\end{array}\right]^{\mathrm{T}} \text {, } \\
& T_{3}=\left[\begin{array}{ll}
I_{5} & O_{5 \times 3}
\end{array}\right]^{\mathrm{T}} \text {, } \\
& T_{4}=\left[\begin{array}{ll}
I_{4} & O_{4 \times 4}
\end{array}\right]^{\mathrm{T}} \text {, } \\
& T_{5}=\left[\begin{array}{ll}
I_{3} & O_{3 \times 5}
\end{array}\right]^{\mathrm{T}} \text {, } \\
& K_{0}=\left[\begin{array}{llllllll}
3 & -6 & 14 & -2 & 6 & 11 & -12 & -6
\end{array}\right] \text {, } \\
& K_{1}=\left[\begin{array}{lllllll}
6 & -11 & 7 & 2 & 7 & 13 & -5
\end{array}\right], \\
& K_{2}=\left[\begin{array}{llllll}
5 & -11 & 12 & 1 & 5 & 12
\end{array}\right] \text {, } \\
& K_{3}=\left[\begin{array}{lllll}
3 & -7 & 15 & -2 & -4
\end{array}\right], \\
& K_{4}=\left[\begin{array}{llll}
7 & -7 & 8 & -7
\end{array}\right] \text {, } \\
& K_{5}=\left[\begin{array}{lll}
3 & -6 & 9
\end{array}\right] \text {. }
\end{aligned}
$$




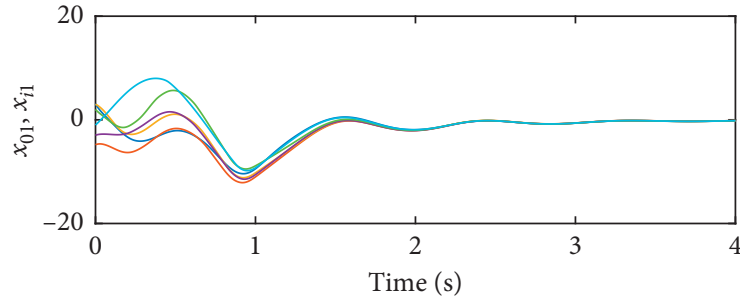

(a)

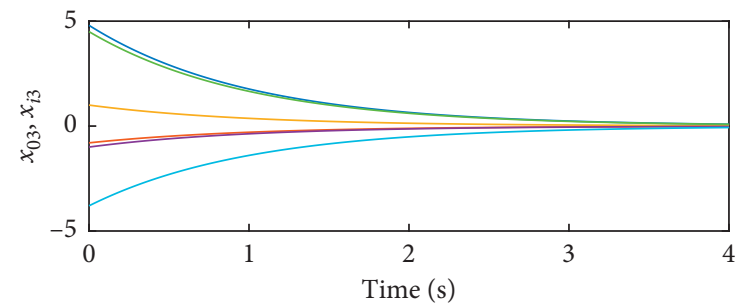

(c)

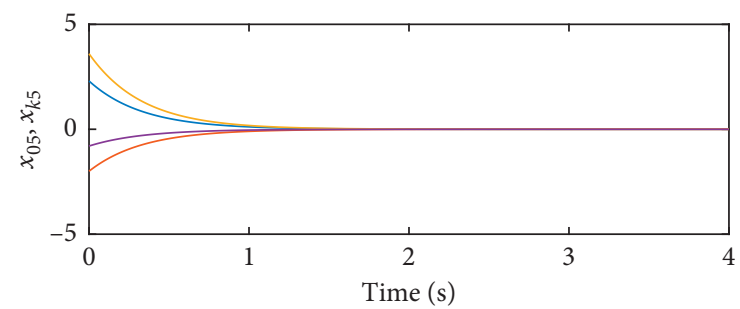

(e)

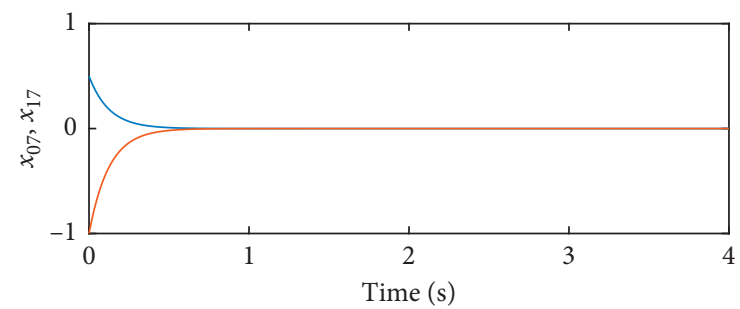

(g)

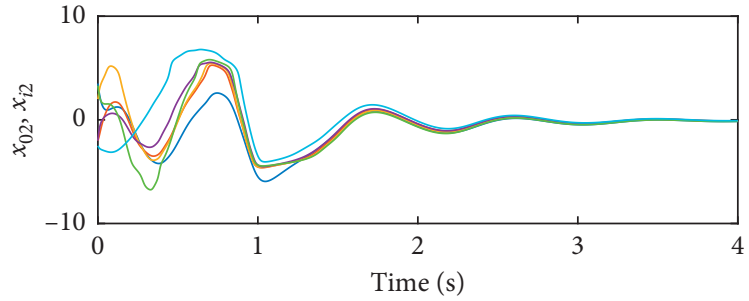

(b)

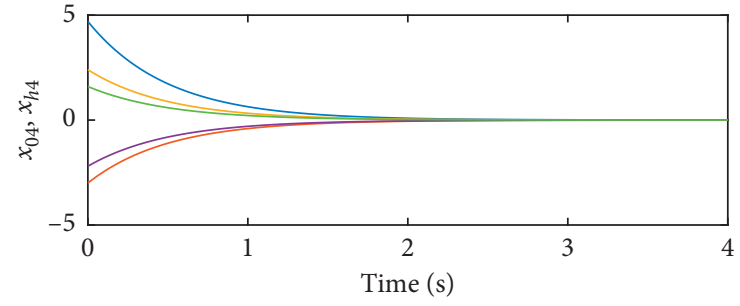

(d)

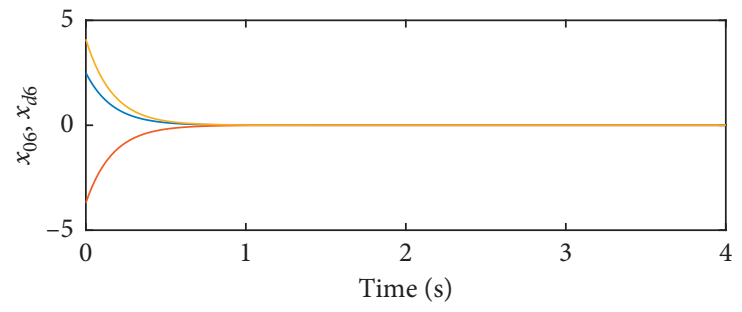

(f)

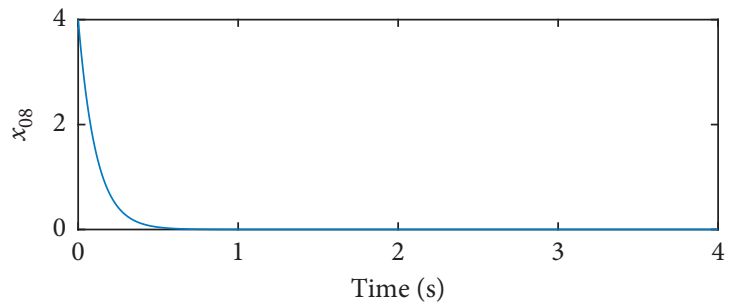

(h)

FIgure 3: Trajectories of the states of leader $x_{0}$ and followers $x_{i}(i=1,2,3,4,5 ; h=1,2,3,4 ; k=1,2,3 ; d=1,2)$.

The nonlinear functions are chosen as

$$
f_{i}=x_{i 1}^{2} \sin x_{i 2} \cos x_{i 3}-0.5 x_{i 2}^{3} \sin x_{i 2}-2 x_{i 3}^{4} \cos x_{i 3} \text {. }
$$

The solutions of the linear matrix inequality (11) are shown as matrices $\bar{K}$ and $P$.

The input bounded signal can be chosen as

$$
s\left(x_{0}, t\right)= \begin{cases}60, & 0<t \leq 1 \\ 0, & 1<t \leq 2 \\ 60, & 2<t \leq 3 \\ 0, & 3<t \leq 4 \\ 60, & 4<t \leq 5\end{cases}
$$

The initial values of the states in the leader and five followers are chosen as

$$
\begin{aligned}
& x_{0}(0)=[3.1,3.5,4.8,4.7,2.3,2.5,0.5,4]^{T}, \\
& x_{1}(0)=[-4.8,-2,-0.8,-3,-2,-3.7,-1]^{T}, \\
& x_{2}(0)=[3,2,1,2.4,3.6,4.1]^{T}, \\
& x_{3}(0)=[-3,-2,-1,-2.2,-0.8]^{T}, \\
& x_{4}(0)=[2,3.3,4.5,1.6]^{T}, \\
& x_{5}(0)=[-1,-2.5,-3.8]^{T} .
\end{aligned}
$$

The initial values of adaptive parameters $\bar{\theta}_{i}(t)$ are given 


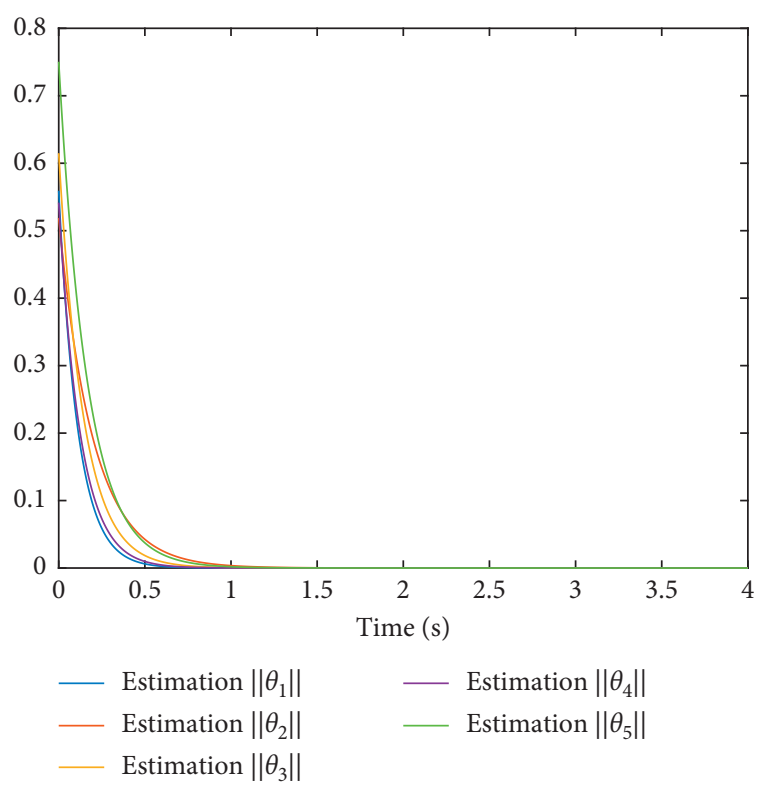

FIgURE 4: Trajectories of the adaptive estimation parameters $\bar{\theta}_{i}(t)$.

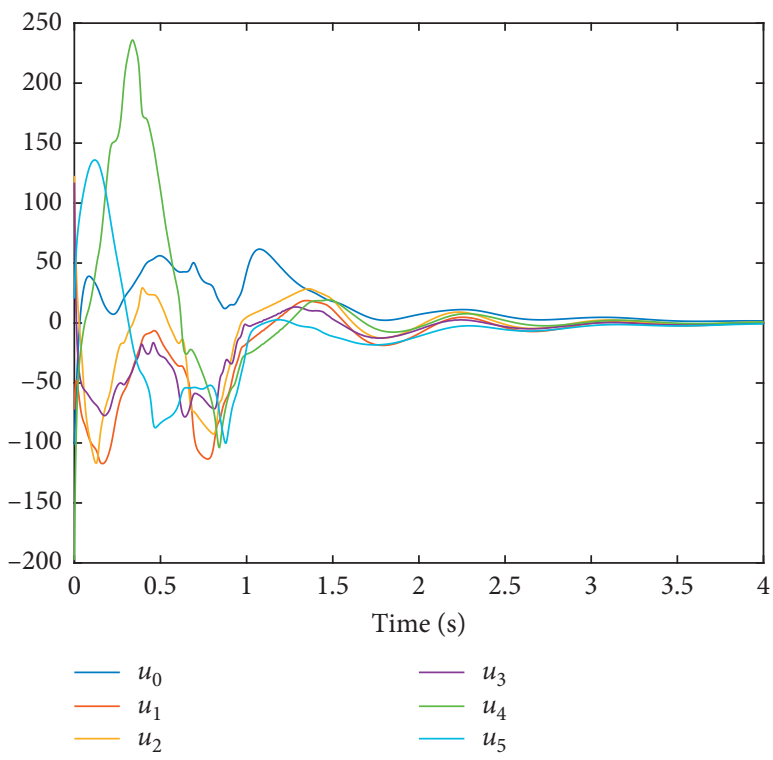

FIgURE 5: Trajectories of the control $u_{0}(t)$ and $u_{i}(t)$.

$$
\begin{aligned}
& \bar{\theta}_{1}(0)=[0.13,0.22,0.10,0.23,0.18,0.39]^{T}, \\
& \bar{\theta}_{2}(0)=[0.24,0.26,0.11,0.14,0.15,0.30]^{T}, \\
& \bar{\theta}_{3}(0)=[0.16,0.29,0.17,0.21,0.35,0.27]^{T}, \\
& \bar{\theta}_{4}(0)=[0.20,0.21,0.11,0.31,0.29,0.13]^{T}, \\
& \bar{\theta}_{5}(0)=[0.34,0.29,0.38,0.26,0.35,0.17]^{T} .
\end{aligned}
$$

The parameters in the adaptive law (12) are chosen as

$$
\begin{aligned}
\kappa_{\theta i} & =\left[\begin{array}{lllll}
9 & 5 & 7 & 8 & 6
\end{array}\right], \\
\rho_{\theta i} & =\left[\begin{array}{lllll}
0.003 & 0.002 & 0.002 & 0.003 & 0.001
\end{array}\right] .
\end{aligned}
$$

The simulation results of the leader system and follower systems are shown as Figures 3-5.

As shown in Figure 3, although the dimensions of leader system and follower systems are nonidentical, the trajectories of $x_{i}$ in follower systems can synchronize to the state of $x_{0}$ in leader system with the proposed distributed fuzzy adaptive control, and it can reach a consistent state in a relatively fast time. Similarly, the norm of adaptive estimated parameters is converged to a small zero field in Figure 4, which can be updated online automatically with the given adaptive laws. From Figure 5, it is shown that the time responses of corresponding control are UUB. Finally, it is concluded that UUB of all signals in the closed-loop system can be guaranteed in Figures 3-5, and the consensus of leader-follower system can be realized by the proposed distribute fuzzy adaptive control with similar parameters whether the leader system and follower systems have the identical or nonidentical dimensions.

\section{Conclusion}

The consensus problem of leader-follower multiagent systems with different dimensions has been considered in this paper. For the unknown nonlinear functions in systems, FLSs are applied to approximate the unknown nonlinear functions, and then a distributed fuzzy adaptive control based on similar condition is designed. With the proposed fuzzy adaptive control, the states of each follower system can stably track the states of the leader system, and it is proved that all signals in the closed-loop system are UUB. The designed method has been verified by a simulation example.

\section{Data Availability}

The data used to support the findings of this study are available from the corresponding author upon request.

\section{Conflicts of Interest}

The authors declare that they have no conflicts of interest.

\section{Acknowledgments}

This work was supported by the National Natural Science Foundation of China (Grant nos. 51875457 and 61903298), Shaanxi Provincial Department of Science and Technology Key Project in the Field of Industry (2018ZDXM-GY-039), and National Natural Science Foundation of Shaanxi (Grant no. 2019JQ-341).

\section{References}

[1] S. D. J. McArthur, E. M. Davidson, V. M. Catterson et al., "Multi-agent systems for power engineering applications-part I: concepts, approaches, and technical challenges," IEEE Transactions on Power Systems, vol. 22, no. 4, pp. 1743-1752, 2007.

[2] S. D. J. McArthur, E. M. Davidson, V. M. Catterson et al., "Multi-agent systems for power engineering applications-part II: technologies, standards, and tools for building 
multi-agent systems," IEEE Transactions on Power Systems, vol. 22, no. 4, pp. 1753-1759, 2007.

[3] T. Li and J.-F. Zhang, "Asymptotically optimal decentralized control for large population stochastic multiagent systems," IEEE Transactions on Automatic Control, vol. 53, no. 7, pp. 1643-1660, 2008.

[4] Z. Peng, D. Wang, H. Zhang, and G. Sun, "Distributed neural network control for adaptive synchronization of uncertain dynamical multiagent systems," IEEE Transactions on Neural Networks and Learning Systems, vol. 25, no. 8, pp. 1508-1519, 2014.

[5] A. Howard, L. E. Parker, and G. S. Sukhatme, "Experiments with a large heterogeneous mobile robot team: exploration, mapping, deployment and detection," International Journal of Robotics Research, vol. 25, no. 5-6, pp. 431-447, 2006.

[6] S.-J. Chung, J.-J. E. Slotine, Cooperative robot control and concurrent synchronization of Lagrangian systems," IEEE Transactions on Robotics, vol. 25, no. 3, pp. 686-700, 2009.

[7] M. Rehan, A. Jameel, and C. K. Ahn, "Distributed consensus control of one-sided Lipschitz nonlinear multiagent systems," IEEE Transactions on Systems, Man, and Cybernetics: Systems, vol. 48, no. 8, pp. 1297-1308, 2018.

[8] F. Xiao and T. Chen, "Adaptive consensus in leader-following networks of heterogeneous linear systems," IEEE Transactions on Control of Network Systems, vol. 5, no. 3, pp. 1169-1176, 2018.

[9] J. Fu, G. Wen, W. Yu, T. Huang, and J. Cao, "Exponential consensus of multiagent systems with Lipschitz nonlinearities using sampled-data information," IEEE Transactions on Circuits and Systems I: Regular Papers, vol. 65, no. 12, pp. 4363-4375, 2018.

[10] J. Qin and C. Yu, "Exponential consensus of general linear multi-agent systems under directed dynamic topology," Automatica, vol. 50, no. 9, pp. 2327-2333, 2014.

[11] L.-X. Wang and J. M. Mendel, "Fuzzy basis functions, universal approximation, and orthogonal least-squares learning," IEEE Transactions on Neural Networks, vol. 3, no. 5, pp. 807-814, 1992.

[12] C. Yang, J. Luo, Y. Pan, Z. Liu, and C.-Y. Su, "Personalized variable gain control with tremor attenuation for robot teleoperation," IEEE Transactions on Systems, Man, and Cybernetics: Systems, vol. 48, no. 10, pp. 1759-1770, 2018.

[13] L. Kong, W. He, C. Yang, Z. Li, and C. Sun, "Adaptive fuzzy control for coordinated multiple robots with constraint using impedance learning," IEEE Transactions on Cybernetics, vol. 49, no. 8, pp. 3052-3063, 2019.

[14] Z. G. Hou, L. Cheng, and M. Tan, "Decentralized robust adaptive control for the multiagent system consensus problem using neural networks," IEEE Transactions on Systems, Man, and Cybernetics, Part B (Cybernetics), vol. 39, no. 3, pp. 636-647, 2009.

[15] W. Ouyang, W. Liang, C. Li, H. Zheng, Q. Ren, and P. Li, "Steering motion control of a snake robot via a biomimetic approach," Frontiers of Information Technology \& Electronic Engineering, vol. 20, no. 1, pp. 32-44, 2019.

[16] C. Yang, Y. Jiang, J. Na, Z. Li, L. Cheng, and C.-Y. Su, "Finitetime convergence adaptive fuzzy control for dual-arm robot with unknown kinematics and dynamics," IEEE Transactions on Fuzzy Systems, vol. 27, no. 3, pp. 574-588, 2019.

[17] W. He, L. Kong, Y. Dong, Y. Yu, C. Yang, and C. Sun, "Fuzzy tracking control for a class of uncertain MIMO nonlinear systems with state constraints," IEEE Transactions on Systems, Man, and Cybernetics: Systems, vol. 49, no. 3, pp. 543-554, 2019.
[18] C. Yang, X. Wang, L. Cheng, and H. Ma, "Neural-learningbased telerobot control with guaranteed performance," IEEE Transactions on Cybernetics, vol. 47, no. 10, pp. 3148-3159, 2017.

[19] Y. Li, C. Yang, W. Yan, R. Cui, and A. Annamalai, "Admittance-based adaptive cooperative control for multiple manipulators with output constraints," IEEE Transactions on Neural Networks and Learning Systems, vol. 30, no. 12, pp. 3621-3632, 2019.

[20] G. Peng, C. Yang, W. He, and C. L. P. Chen, "Force sensorless admittance control with neural learning for robots with actuator saturation," IEEE Transactions on Industrial Electronics, vol. 67, no. 4, pp. 3138-3148, 2020.

[21] C. Yang, C. Chen, W. He, R. Cui, and Z. Li, "Robot learning system based on adaptive neural control and dynamic movement primitives," IEEE Transactions on Neural Networks and Learning Systems, vol. 30, no. 3, pp. 777-787, 2019.

[22] Q. Shen, P. Shi, and Y. Shi, "Distributed adaptive fuzzy control for nonlinear multiagent systems via sliding mode observers," IEEE Transactions on Cybernetics, vol. 46, no. 12, pp. 30863097, 2016.

[23] Y.-X. Li, G.-H. Yang, and S. Tong, "Fuzzy adaptive distributed event-triggered consensus control of uncertain nonlinear multiagent systems," IEEE Transactions on Systems, Man, and Cybernetics: Systems, vol. 49, no. 9, pp. 1777-1786, 2019.

[24] L. Cao, H. Li, G. Dong, and R. Lu, "Event-triggered control for multiagent systems with sensor faults and input saturation," IEEE Transactions on Systems, Man, and Cybernetics: Systems, pp. 1-12, 2019.

[25] Q. Zhou, W. Wang, H. Liang, M. V. Basin, and B. Wang, "Observer-based event-triggered fuzzy adaptive bipartite containment control of multi-agent systems with input quantization," IEEE Transactions on Fuzzy Systems, pp. 1-13, 2019.

[26] H. Kim, H. Shim, and J. H. Seo, "Output consensus of heterogeneous uncertain linear multi-agent systems," IEEE Transactions on Automatic Control, vol. 56, no. 1, pp. 200-206, 2011.

[27] W. Hu, L. Liu, and G. Feng, "Output consensus of heterogeneous linear multi-agent systems by distributed eventtriggered/self-triggered strategy," IEEE Transactions on Cybernetics, vol. 47, no. 8, pp. 1914-1924, 2017.

[28] Y. Lv, Z. Li, Z. Duan, and G. Feng, "Novel distributed robust adaptive consensus protocols for linear multi-agent systems with directed graphs and external disturbances," International Journal of Control, vol. 90, no. 2, pp. 137-147, 2017.

[29] C. L. P. Chen, C.-E. Ren, and T. Du, "Fuzzy observed-based adaptive consensus tracking control for second-order multiagent systems with heterogeneous nonlinear dynamics," IEEE Transactions on Fuzzy Systems, vol. 24, no. 4, pp. 906915, 2016

[30] C.-E. Ren, L. Chen, and C. L. P. Chen, “Adaptive fuzzy leaderfollowing consensus control for stochastic multiagent systems with heterogeneous nonlinear dynamics," IEEE Transactions on Fuzzy Systems, vol. 25, no. 1, pp. 181-190, 2017.

[31] Z. Li, Z. Duan, and F. L. Lewis, "Distributed robust consensus control of multi-agent systems with heterogeneous matching uncertainties," Automatica, vol. 50, no. 3, pp. 883-889, 2014.

[32] Y. Lv, Z. Li, and Z. Duan, "Distributed PI control for consensus of heterogeneous multiagent systems over directed graphs," IEEE Transactions on Systems, Man, and Cybernetics: Systems, vol. 50, no. 4, pp. 1602-1609, 2020.

[33] C. D. Araújo and J. D. Castro, "Application of power system stabilisers in a plant with identical units," IEEE Proceedings $C$ 
(Generation, Transmission and Distribution), vol. 138, no. 1, pp. 11-18, 1991.

[34] L. Bakule and J. Lunze, "Decentralized design of feedback control for large-scale systems," Kybernetika, vol. 24, no. 8 , pp. 1-3, 1988.

[35] Y.-H. Wang and S.-Y. Zhang, "Robust control for nonlinear similar composite systems with uncertain parameters," IEE Proceedings-Control Theory and Applications, vol. 147, no. 1, pp. 80-86, 2000.

[36] X.-G. Yan and G.-Z. Dai, "Decentralized output feedback robust control for nonlinear large-scale systems," Automatica, vol. 34, no. 11, pp. 1469-1472, 1998.

[37] X.-G. Yan, J. Lam, and G.-Z. Dai, "Decentralized stabilization for nonlinear similar composite systems with uncertainty," IFAC Proceedings Volumes, vol. 32, no. 2, pp. 3444-3449, 1999.

[38] Y. Wang, Y. Fan, Q. Wang, and Y. Zhang, "Stabilization and synchronization of complex dynamical networks with different dynamics of nodes via decentralized controllers," IEEE Transactions on Circuits and Systems I: Regular Papers, vol. 59, no. 8, pp. 1786-1795, 2012.

[39] P. Wang and Y. Jia, "Robust $H^{\infty}$ containment control for uncertain multi-agent systems with inherent nonlinear dynamics," International Journal of Systems Science, vol. 47, no. 5, pp. 1073-1083, 2016. 